\title{
Muon-electron scattering at next-to-leading order accuracy
}

\author{
Carlo M. Carloni Calame ${ }^{1, *}$, Mauro Chiesa ${ }^{2}$, Guido Montagna ${ }^{3,1}$, Oreste Nicrosini ${ }^{1}$, and \\ Fulvio Piccinini ${ }^{1}$ \\ ${ }^{1}$ INFN, Sezione di Pavia, via A. Bassi 6, Pavia 27100 (Italy) \\ ${ }^{2}$ Institut für Theoretische Physik und Astrophysik, Julius-Maximilians-Universität Würzburg, Emil- \\ Hilb-Weg 22, Würzburg D-97074 (Germany) \\ ${ }^{3}$ Dipartimento di Fisica, Università di Pavia, via A. Bassi 6, Pavia 27100 (Italy)
}

\begin{abstract}
The next-to-leading order electro-weak radiative corrections to the $\mu^{ \pm} e^{-} \rightarrow \mu^{ \pm} e^{-}$process are reviewed and their relevance is discussed for the MUonE experiment, proposed at CERN. The aim of MUonE is the high precision measurement of the QED running coupling constant in the space-like region, from which the full hadronic contribution can be extracted and used to provide a new and independent determination of the leading-order hadronic correction to the muon $g-2$. In this context, the required accuracy demands that radiative corrections are accounted for at the highest level of precision and implemented into a Monte Carlo event generator for data analysis. The first step towards the final goal of theoretical precision, which will require the full set of NNLO corrections and resummation of higher orders, is the inclusion of NLO electro-weak corrections.
\end{abstract}

\section{Introduction}

The MUonE experiment, proposed to run at CERN, aims at measuring with high precision the running of the QED fine-structure constant $\alpha\left(q^{2}\right)$ in the space-like region $\left(q^{2}<0\right)$ [13]. As firstly put forward in [4], by extracting the leading hadronic part of the running of $\alpha$ at negative $q^{2}$, an independent prediction of the hadronic leading-order (HLO) correction to the muon $g-2\left(a_{\mu}^{\mathrm{HLO}}\right)$ can be provided. Since the standard $a_{\mu}^{\mathrm{HLO}}$ calculations, exploiting a dispersive integral over $\sigma\left(e^{+} e^{-} \rightarrow\right.$ hadrons $)$, currently give a large fraction of the theoretical uncertainty in the prediction of the muon anomaly, such independent estimate can shed light on the long-standing and persistent 3-4 $\sigma$ discrepancy between the Standard Model (SM) prediction of the muon $g-2$ and its most precise experimental measurement [5-10].

The MUonE experiment plans to scatter $150 \mathrm{GeV}$ muons off electrons of a low- $Z$ target $\left(B e\right.$ or $C$ ). By studying the elastic $t$-channel scattering process $\mu^{ \pm} e^{-} \rightarrow \mu^{ \pm} e^{-}$at $\sqrt{s} \sim 0.406 \mathrm{GeV}$, a direct measurement of the hadronic correction to the vacuum polarization $\Delta \alpha_{\text {had }}(t)$ can be performed and used to calculate $a_{\mu}^{\mathrm{HLO}}$. The CERN M2 facility provides a high-intensity muon beam with the correct properties which would allow MUonE to reach a $0.3 \%$ statistical error on $a_{\mu}^{\mathrm{HLO}}$ in two years of data taking. In order for this to happen, the systematic uncertainties must be kept under control at the $10 \mathrm{ppm}$ level, which is the most demanding challenge of the proposed experiment.

\footnotetext{
*e-mail: carlo.carloni.calame@pv.infn.it
} 
The 10ppm precision goal in the measurement of the cross section requires that also the theoretical simulations and calculations needed for data analysis reach the same level of accuracy. In turn this requires the inclusion of radiative corrections (RCs), in particular QED $\mathrm{RCs}$, at the highest level of precision. In the last few years a constant effort has been employed for the calculation of RCs at NLO [11] and important progresses have been made towards the calculations of QED RCs at NNLO [12-15]. In the near future, the ultimate Monte Carlo (MC) tool for data analysis must include all these ingredients together with resummation of higher-order QED corrections and its development is under consideration.

In the following, largely based on the results of Ref. [11], we sketch an overview of the NLO calculation, which is now implemented into a Monte Carlo event generator (EG) used by the MUonE collaboration, and we show some phenomenological results.

\section{Calculation details}

The first step towards the control of the theoretical cross section at the 10ppm level is the calculation of the NLO RCs in the SM. Without entering the full details, which are discussed at length in [11], we report here the principal features of the calculation:

1. tree-level, one-loop virtual and real photon emission QED amplitudes are manipulated with the help of FORM $[16,17]$. One loop scalar functions and tensor coefficients are numerically evaluated with the library LoopTools $[18,19]$ and cross-checked against Collier [20], finding up-to-digit agreement;

2. following standard and common techniques, infra-red (IR) divergencies are treated introducing a vanishingly small photon mass $\lambda$ and a $[2 \rightarrow 2] /[2 \rightarrow 3]$ phase space splitting is performed introducing a fictitious soft/hard photon energy separator $\omega_{s}$. The independence from $\lambda$ and $\omega_{s}$ of any observable and cross-section is verified with high numerical accuracy;

3. muon and electron masses $\left(m_{\mu}, m_{e}\right)$ must be kept everywhere, i.e. any expansion in $m_{\mu}$ or $m_{e}$ would result in a too large theoretical error;

4. also the impact of purely weak NLO RCs are studied and calculated with the help of the program Recola [21]. It turns out that at NLO they can be neglected, their size being well below the 10ppm level. Nevertheless, tree-level $Z$ exchange diagrams must be taken into account at this level of accuracy.

The calculation is implemented into a fully exclusive MC EG, which is a derivation of the widely used generator BabaYaga@NLO [22]. Samples of events produced by the NLO generator are extensively used by the MUonE collaboration for feasibility studies and simulation of the experiment.

\section{Numerical results}

We remind that in the MUonE setup $s \sim 0.164 \mathrm{GeV}^{2}$ and that the $t$ Mandelstam variable (for elastic events) approximately spans the range $\left[-0.14,-1 \cdot 10^{-3}\right] \mathrm{GeV}^{2}$ if the outgoing $e^{-}$is required to have an energy $E_{e}>0.2 \mathrm{GeV}$. Correspondingly, the outgoing muon angle $\theta_{\mu}$ is lower than $\sim 5 \mathrm{mrad}$ and the electron angle $\theta_{e}$ lower than $\sim 73 \mathrm{mrad}$.

For the sake of presenting realistic phenomenological results, we consider four different experimental selection criteria:

1. $\theta_{e}, \theta_{\mu}<100 \mathrm{mrad}$ and $E_{e}>0.2 \mathrm{GeV}$; 
2. $\theta_{e}, \theta_{\mu}<100 \mathrm{mrad}$ and $E_{e}>1 \mathrm{GeV}$. With respect to Setup 1, a higher electron energy threshold is imposed to focus on the region where $\Delta \alpha_{\text {had }}(t)$ is larger;

3. the same criteria as in Setup 1, with an additional acoplanarity cut, applied to partially remove radiative events and thus enhancing the fraction of elastic events. We require acoplanarity $\equiv\left|\pi-\left(\phi_{e}-\phi_{\mu}\right)\right| \leq 3.5$ mrad for the sake of illustration;

4. the same criteria as in Setup 2, with the additional acoplanarity cut as in Setup 3.
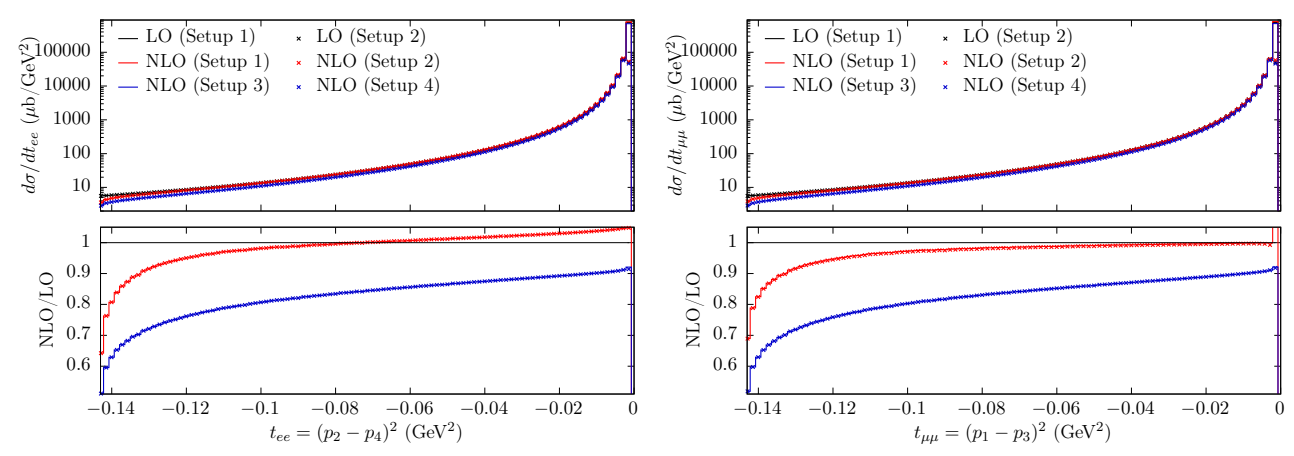

Figure 1. $t_{e e}$ and $t_{\mu \mu}$ distributions and NLO corrections for the $\mu^{+} e^{-} \rightarrow \mu^{+} e^{-}$process. See the text for details.

In Fig. 1 (from [11]) the impact of QED NLO RCs is shown on the distributions of the $t_{e e}$ and $t_{\mu \mu}$ Mandelstam variables, where $t_{e e(\mu \mu)}$ is the $t$ variable calculated on the electron (muon) current ${ }^{1}$. In the figure, the upper panels show the absolute differential cross section and the lower panels the ratio between the NLO corrected differential cross section and the LO one. We notice that QED NLO effects lie in the 10-40\% range, increasing in size as $E_{e}$ is increased.
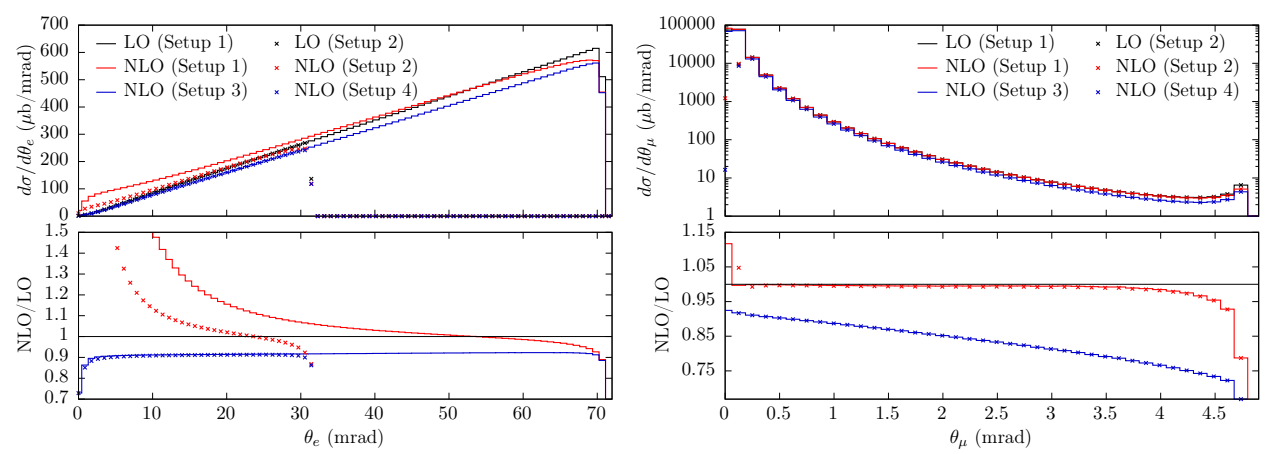

Figure 2. $\theta_{e}$ and $\theta_{\mu}$ distributions and NLO corrections for the $\mu^{+} e^{-} \rightarrow \mu^{+} e^{-}$process. See the text for details.

Analogously, Fig. 2 (from [11]) shows the impact of NLO RCs on the $\theta_{e}$ and $\theta_{\mu}$ variables, which are the primary observables measured in the MUonE experiment. We would like

\footnotetext{
${ }^{1}$ Notice that beyond $\mathrm{LO} t_{e e}$ and $t_{\mu \mu}$ do not coincide anymore.
} 
to stress that on $\theta_{e}$ NLO RCs can become quite sizable but the acoplanarity elasticity cut makes them more moderate: the large effect in the absence of elasticity cut can be ascribed to radiative events $\mu^{+} e^{-} \rightarrow \mu^{+} e^{-} \gamma$, when large-angle (soft) electrons are scattered in the low $\theta_{e}$ region due to the emission of an hard photon.
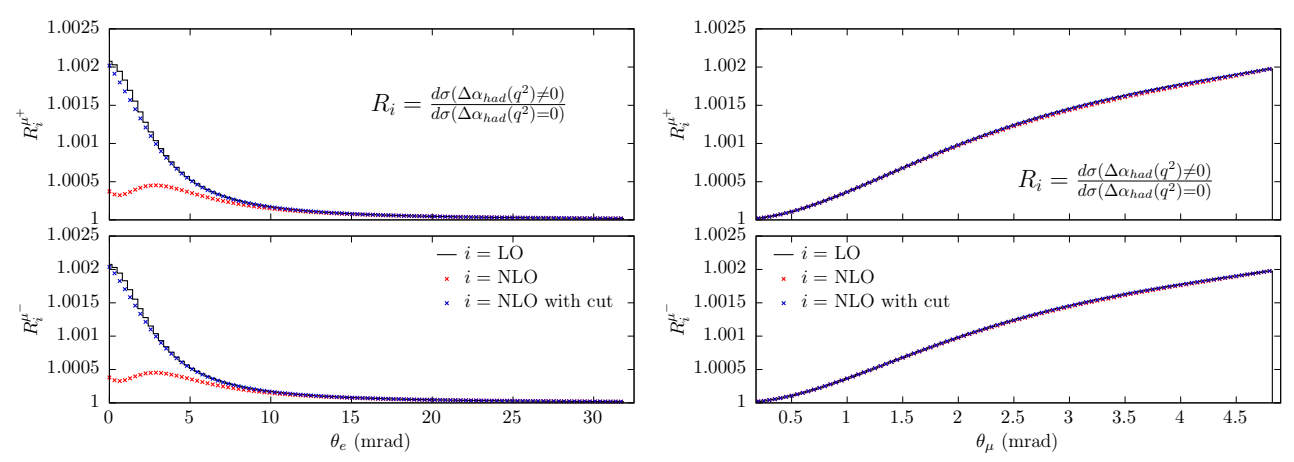

Figure 3. Signal $R$ ratio in different Setups. See the text for details.

Another instructive and important result is shown in Fig. 3 (from [11]). In the figures, for the $\theta_{e}$ and $\theta_{\mu}$ observables the $R$ quantity is defined as the ratio

$$
R=\frac{d \sigma\left(\Delta \alpha_{\text {had }}\left(q^{2}\right) \neq 0\right)}{d \sigma\left(\Delta \alpha_{\text {had }}\left(q^{2}\right)=0\right)} .
$$

In words, $R$ shows the effect on the given observable of the hadronic part of the QED running coupling constant, which is the quantity that MUonE aims to extract from data. The left-side plots show that on $\theta_{e}$ the LO "signal" (black histograms) is highly reduced at NLO if no elasticity cuts are applied (red dots) and recovered when they are applied (blue dots). On the other hand, in the right-side plots, the $\theta_{\mu}$ variable looks much more insensitive to both inclusion of NLO effects and application of experimental cuts.

We would like also to stress that from Fig. 3 it can be deduced that the quantity to be extracted from data, $\Delta \alpha_{\text {had }}\left(q^{2}\right)$, is a $O\left(10^{-3}\right)$ effect on the differential cross-sections.

\section{Conclusions and outlook}

We reported the calculation of the NLO radiative corrections to the processes $\mu^{ \pm} e^{-} \rightarrow \mu^{ \pm} e^{-}$ and their implementation into a Monte Carlo event generator, needed in view of the proposed MUonE experiment. MUonE aims at a high-precision direct measurement of the leadingorder hadronic correction to the running of the fine-structure constant with the goal of providing a new independent prediction of the leading-order hadronic part of the muon anomalous magnetic moment $a_{\mu}^{\mathrm{HLO}}$. This will possibly shed light on the current discrepancy between the SM prediction of the muon $g-2$ and its experimental value. The discrepancy could also increase in size to 6-7 $\sigma$ after the FNAL [23, 24] and the future J-PARC [25] $g-2$ experiments will make their results public.

The main MUonE challenge will be keeping the systematic uncertainties on the differential cross-section measurements at the $10 \mathrm{ppm}$ level. This requires that also theoretical calculations and tools must match the same precision: a careful inclusion of all the important radiative corrections is thus mandatory. In order to reach such a theoretical accuracy, both 
fixed-order QED corrections up to NNLO and resummation of higher-orders, and their consistent combination, will be necessary. A first step towards the final goal is the calculation of NLO corrections within the SM and the close scrutiny of the different sources of effects (finite mass corrections, gauge-invariant subsets, etc.). Their implementation into a Monte Carlo generator is of utmost importance in this phase of feasibility studies and design of the MUonE experiment.

The extension of the NLO Monte Carlo to include the complete set of the required corrections is now under development.

\section{Acknowledgments}

C.M.C.C. would like to warmly thank prof. Simon Eidelman and the PhiPsi 2019 Local Organizing Committee for the kind invitation and for organizing a successful and friendly workshop.

\section{References}

[1] G. Abbiendi et al., Eur. Phys. J. C77, 139 (2017), 1609. 08987

[2] U. Marconi, these proceedings (2019)

[3] M. Passera, these proceedings (2019)

[4] C.M. Carloni Calame, M. Passera, L. Trentadue, G. Venanzoni, Phys. Lett. B746, 325 (2015), 1504.02228

[5] G. Colangelo, these proceedings (2019)

[6] F. Jegerlehner, Springer Tracts Mod. Phys. 274, pp. 1 (2017)

[7] F. Jegerlehner, Acta Phys. Polon. B49, 1157 (2018), 1804 . 07409

[8] M. Davier, A. Hoecker, B. Malaescu, Z. Zhang, Adv. Ser. Direct. High Energy Phys. 26, 129 (2016)

[9] A. Keshavarzi, D. Nomura, T. Teubner, Phys. Rev. D97, 114025 (2018), 1802.02995

[10] G.W. Bennett et al. (Muon g-2), Phys. Rev. D73, 072003 (2006), hep-ex/0602035

[11] M. Alacevich, C.M. Carloni Calame, M. Chiesa, G. Montagna, O. Nicrosini, F. Piccinini, JHEP 02, 155 (2019), 1811.06743

[12] P. Mastrolia, M. Passera, A. Primo, U. Schubert, JHEP 11, 198 (2017), 1709. 07435

[13] S. Di Vita, S. Laporta, P. Mastrolia, A. Primo, U. Schubert (2018), 1806.08241

[14] M. Fael, JHEP 02, 027 (2019), 1808.08233

[15] M. Fael, M. Passera (2019), 1901.03106

[16] J. Kuipers, T. Ueda, J.A.M. Vermaseren, J. Vollinga, Comput. Phys. Commun. 184, 1453 (2013), 1203.6543

[17] B. Ruijl, T. Ueda, J. Vermaseren (2017), 1707.06453

[18] T. Hahn, PoS ACAT2010, 078 (2010), 1006.2231

[19] T. Hahn, M. Perez-Victoria, Comput. Phys. Commun. 118, 153 (1999), hep-ph/9807565

[20] A. Denner, S. Dittmaier, L. Hofer, Comput. Phys. Commun. 212, 220 (2017), 1604.06792

[21] S. Actis, A. Denner, L. Hofer, J.N. Lang, A. Scharf, S. Uccirati, Comput. Phys. Commun. 214, 140 (2017), 1605.01090

[22] G. Balossini, C.M. Carloni Calame, G. Montagna, O. Nicrosini, F. Piccinini, Nucl. Phys. B758, 227 (2006), hep-ph/0607181

[23] J. Grange et al. (Muon g-2) (2015), 1501.06858 
[24] A. Keshavarzi, The Muon g - 2 experiment at Fermilab, in International Workshop on e+e-collisions from Phi to Psi (PhiPsi19) Novosibirsk, Russian Federation, February 25-March 1, 2019 (2019), 1905.00497

[25] T. Mibe (J-PARC g-2), Nucl. Phys. Proc. Suppl. 218, 242 (2011) 